http://jmscr.igmpublication.org/home/ ISSN (e)-2347-176x ISSN (p) 2455-0450 crossref DOI: https://dx.doi.org/10.18535/jmscr/v10i1.02

\title{
Study of Liver Abscess Drainage by Needle Aspiration v/s Pigtail Catheter Drainage in a Tertiary Care Hospital
}

\author{
Dr Raj Depani ${ }^{1}$, Dr Tirth Bharat Shah ${ }^{2}$, Dr Shubham Patel ${ }^{3}$, Dr Sharad M Patel ${ }^{4}$ \\ ${ }^{1,2,3}$ Resident Doctor, Department of General Surgery, Smt SCL HOSPITAL, NHL MMC, Ahmedabad \\ ${ }^{4}$ Professor, Department of General Surgery, Smt SCL HOSPITAL, NHL MMC, Ahmedabad
}

\begin{abstract}
Aim: To compare effectiveness and outcome of USG guided needle aspiration and percutaneous catheter drainage in patients diagnosed with liver abscess.

Methods: This is a prospective observational study of liver abscess drainage over the period of 28 months from 1st June 2019 to 31 st Oct 2021 at the department of General Surgery, Smt. SCL General Hospital, Ahmedabad.

Study consists of 130 patients, presented in the outpatient and emergency department at the hospital, selected by random sampling method and diagnosed by ultrasonography.

Results: Majority (63.8\%) patients treated by percutaneous aspiration had a mean liver abscess cavity of $6 \mathrm{~cm}$ size $158 \mathrm{cc}$. Pigtail catheter drain was placed in $25.38 \%$ patients having large abscess with average size $7.5 \mathrm{~cm}$ and $565 \mathrm{cc}$ volume. Recurrence was seen in $24 \%$ patients who were percutaneously aspirated whereas in catheter drainage, recurrence rate was 9\%. Average duration of hospital stay was 7.83 days \& 9.71 days in patients treated by percutaneous aspiration and pigtail catheterisation respectively.

Conclusions: Percutaneous needle aspiration is preferred in patients with single or multiple liver abscess with cavity size of $>5 \mathrm{~cm}$ and $>50 \mathrm{cc}$ volume. In abscess with cavity size $>10 \mathrm{~cm}$ and single cavity in adults can be treated with Percutaneous catheter drainage.

Keywords: Liver abscess, Pigtail Catheter drainage, Needle aspiration.
\end{abstract}

\section{Introduction}

The first description of Liver abscess is credited to Hippocrates in the year 4000 BC. It was mentioned in association with blood and mucus diarrhoeal stools. Liver abscess was first drained in the Hippocratic era and successfully practiced the draining of pus.

Liver abscesses are purulent collections in the liver parenchyma that result from bacterial, fungal, parasitic or mixed infection.
Pyogenic liver abscess (PLA) may be defined as a solitary or multiple collections of pus within the liver due to bacterial infections commonly Streptococcus milleri, Escherichia coli, and other enteric organisms such as S. faecalis, Klebsiella and Proteus vulgaris.

Amoebic liver abscess is the most frequent extraintestinal manifestation of Entamoeba histolytica infection, which enters the portal venous system from the colon. 
Fungal liver abscesses have recently been recognized with increased frequency in immunosuppressed states secondary to chemotherapy, infection with the human immunodeficiency virus (HIV), and in patients with haematological malignancies during periods of neutropenic resolution.

The clinical presentation of both the types may be elusive with combination of fever, right upper quadrant pain and hepatomegaly with or without jaundice.

Liver abscesses continue to be an important cause of morbidity and mortality in tropical countries. However, recent advances in interventional radiology, intensive care, progress in antibiotic therapy, and use of sonography and computerized tomography scanning of the abdomen have led to early diagnosis and treatment of patients with liver abscess, thus improving the patient outcome.

Previously liver abscess was regarded as a high morbidity disease requiring open surgical drainage, with mortality rates between $9 \%$ and $80 \%$. If untreated, it was uniformly fatal. In the last quarter of a century we have witnessed a major paradigm shift in the management of pyogenic hepatic abscesses/amoebic liver abscesses, with a concomitant decrease in mortality to 5-30\%. Percutaneous drainage of liver abscess has been an important advancement and is traditionally used in the treatment of both types of liver abscesses.

\section{Methods}

This is a prospective observational study of liver abscess drainage over the period of 28 months from 1st June 2019 to 31st Oct 2021 at the department of General Surgery, Smt. SCL General Hospital, Ahmedabad.

Study consists of 130 patients, presented in the outpatient and emergency department at the hospital, selected by random sampling method and diagnosed by ultrasonography.

\section{Inclusion Criteria}

All cases of liver abscess diagnosed clinically and radiological investigations during study period randomly allocated in study.

\section{Exclusion Criteria}

- Patients below 3 and above 70 years of age

- Patients requiring exploratory laparotomy

- Traumatic liver abscess

- Liver malignancies

- Chronic liver disease

Cases with abscess cavity $<5 \mathrm{~cm}$ or $<50 \mathrm{cc}$ volume were treated by medical therapy alone. Failures to relieve symptoms within 2 to 3 days were treated by percutaneous aspiration. Those with abscess cavity $>5 \mathrm{~cm}$ or $>50 \mathrm{cc}$ volume were treated by Percutaneous aspiration. Bilateral abscess cavities that were small and multiple were managed by medical therapy and when any one of the cavity size $>5 \mathrm{~cm}$ or $>50 \mathrm{cc}$ volume was managed by Percutaneous aspiration. Abscess cavities restricted to the left lobe were treated by drug therapy if they were multiple and cavity size $<5 \mathrm{~cm}$. Those with single abscess cavity size $>10$ $\mathrm{cm}$ in adults and $>5 \mathrm{~cm}$ and $<10 \mathrm{yr}$ age were treated with Percutaneous catheter drainage. In case of multiple cavities if the size of any cavity $>10 \mathrm{~cm}$ were treated by Percutaneous catheter drainage. Those abscess cavities with subcapsular rupture were treated with percutaneous catheter drainage. Abscess cavities with intra-abdominal rupture were managed by open surgical drainage. With all due permission, data was collected from the case papers and hospital records. Statistical analysis was done by appropriate statistical tools.

\section{Results}

\section{Socio Demographic Data}

Out of 130 patients in the study, 99 (76\%) were male and $31(23.8 \%)$ were female. The mean age distribution of the study group is 31.9. The commonest age group for liver abscess was 31-40 years. 
Table 1: Presenting Symptoms

The commonest symptom was abdominal pain seen in $85 \%$ of patients followed by fever in $81.5 \%$, Nausea and vomiting in $35.3 \%$, jaundice and diarrhoea in $10 \%$ patients.

\begin{tabular}{|l|c|}
\hline Symptom & No. of Patients \\
\hline Abdominal pain & $111(85.3 \%)$ \\
\hline Fever & $106(81.5 \%)$ \\
\hline Nausea \& Vomiting & $49(37.7 \%)$ \\
\hline Jaundice & $13(10 \%)$ \\
\hline Diarrhoea & $13(10 \%)$ \\
\hline Cough & $12(9.23 \%)$ \\
\hline Altered sensorium & 0 \\
\hline Signs \\
\hline Abdominal tenderness & $65(50 \%)$ \\
\hline Hepatomegaly & $77(59.2 \%)$ \\
\hline Ascites & $22(16.9 \%)$ \\
\hline Anemia $(\mathrm{Hb}<10 \mathrm{~g} / \mathrm{dl})$ & $32(24.6 \%)$ \\
\hline
\end{tabular}

Table 2 Association

Alcohol is the most important risk factor for liver abscess. $50 \%$ of the patients in the study were alcoholic.

\begin{tabular}{|l|c|}
\hline Associations & No. of Patients \\
\hline Alcohol & $65(50 \%)$ \\
\hline Pulmonary Tuberculosis & $6(4.6 \%)$ \\
\hline Hypertension & $4(3 \%)$ \\
\hline Pancreatitis & $4(3 \%)$ \\
\hline Diabetes type 2 & $2(1.5 \%)$ \\
\hline Pregnancy & $1(0.7 \%)$ \\
\hline
\end{tabular}

Table 3 Anatomical Location of Liver Abscess

\begin{tabular}{|l|c|}
\hline Parameters & No. of Patients \\
\hline Lobe Involved & $101(77.6 \%)$ \\
\hline Right lobe & $19(14.6 \%)$ \\
\hline Left lobe & $10(7.6 \%)$ \\
\hline Both lobes \\
\hline No. Of Abscess Cavity \\
\hline SINGLE \\
\hline MULTIPLE & $80(61.5 \%)$ \\
\hline Type Of Liver Abscess & $50(38.5 \%)$ \\
\hline Amoebic \\
\hline Pyogenic & $53(40.8 \%)$ \\
\hline Mixed & $58(44.6 \%)$ \\
\hline
\end{tabular}

In the study the right lobe was involved in $77.6 \%$ of cases whereas the left lobe and both lobes were involved in $14.6 \%$ and $7.6 \%$ of patients respectively. Single abscess cavity and Multiple abscess cavities were found in $61.5 \%$ and $38.5 \%$ of cases. Prevalence of amoebic liver abscess was $40.8 \%$ and that of pyogenic liver abscess was $44.6 \%$ patients. $7.7 \%$ patients had mixed amoebic and pyogenic abscess. None of the patients with fungal liver abscess isolated.

Table 4: Treatment Analysis

\begin{tabular}{|l|c|c|c|}
\hline Treatment & No. of Patients & Mean Cavity Size (Cm) & Mean Cavity Volume(Cc) \\
\hline Conservative & 09 & 3.8 & 031 \\
\hline Percutaneous Aspiration & 83 & 6 & 158 \\
\hline Pigtail catheter drainage & 33 & 7.5 & 565 \\
\hline Aspiration + Pigtail Drain & 05 & 8.8 & 624 \\
\hline
\end{tabular}

$83(63.8 \%)$ patients treated by percutaneous aspiration had mean liver abscess cavity of $6 \mathrm{~cm}$ size $158 \mathrm{cc}$. Pigtail catheter drain was placed in 33 $(25.38 \%)$ patients having large abscess with

average size $7.5 \mathrm{~cm}$ and $565 \mathrm{cc}$ volume. $5(3.8 \%)$ patients which were treated by aspiration had to eventually undergo placement of pigtail drain as the mean cavity size was $8.8 \mathrm{~cm} 624 \mathrm{cc}$.

Table 5: Outcome And Hospital Stay Duration Compared In Both Treatment Modality

\begin{tabular}{|l|c|c|c|}
\hline Treatment Modality & No. of Patients & $\begin{array}{c}\text { Recurrence } \\
\text { (No. of Patients) }\end{array}$ & $\begin{array}{c}\text { Average Duration of } \\
\text { stay (Days) }\end{array}$ \\
\hline Conservative & 09 & 0 & 5.65 \\
\hline Percutaneous Aspiration & 83 & 20 & 7.83 \\
\hline Pigtail Catheter Drainage & 33 & 03 & 9.71 \\
\hline
\end{tabular}

Recurrence was seen in $24 \%$ patients who were percutaneously aspirated whereas in catheter drainage, recurrence rate was $9 \%$. Average duration of hospital stay was 7.83, 9.71, 5.65 in patients treated by percutaneous aspiration, pigtail catheterisation and conservatively respectively. 


\section{Discussion}

Age distribution was consistent with the previous studies of Sukhjeet Singh et al ${ }^{[1]}$ and Arpit Bansal et $\mathrm{al}^{[2]}$. having a mean age of 31.9 years. The highest incidence of liver abscess was noted in the age group 31-40 years of age (26.9\%) followed by $41-50$ years of age $(15.38 \%)$ in this study. Male to Female patient ratio is 3.19:1. Male preponderance is possibly due to alcohol consumption and portal sepsis.

Fever and abdominal pain over right hypochondrium are the most common manifestations $^{[1,2]}$ of liver abscess. In the present study, these two symptoms of Fever and Abdominal pain occurred in $81.5 \%$ and $85 \%$ patients. Abdominal pain \& fever are due to inflammatory involvement of liver \& small intestine due to pyogenic and amoebic infections. Alcoholism is an important etiological risk factor associated with liver abscess ${ }^{[3]}$. $50 \%$ of the Adult Male cases of the present study were found to be alcoholics.

Right lobe was involved in $77.6 \%$ of cases of the present study. The predilection of liver abscess in the right lobe is because of straight directional flow of portal circulation through portal vein and it receives most of blood draining from the right colon. In the study, the left lobe and both lobes were involved in $14.6 \%$ and $7.6 \%$ of patients. This is compared with the study of Sukhjeet Singh et $\mathrm{al}^{[1]}$ who recorded $78 \%$ involvement in the right lobe, $15 \%$ in left lobe and $7 \%$ in both lobes.

Single abscess cavities were found to be $61.5 \%$ and multiple abscess cavities in $38.5 \%$ of patients of this study. This is compared with the study conducted by Arpit Bansal et al ${ }^{[2]}$ who recorded single abscess in $12.4 \%$ and multiple abscesses in $87.6 \%$. Patients who had multiple small abscesses and single abscess with cavity $<5 \mathrm{~cm}$ size or $<$ $50 \mathrm{cc}$ volume with no any active complaint were successfully managed conservatively. The conservative management was done in $6.9 \%$ of cases.

In $59 \%$ patients who had abscess $>50$ cc or cavity size $>5 \mathrm{~cm}$ were treated with percutaneous aspiration. Patients showed improvements in their symptoms and signs within 24-72 hrs of the aspiration.

According to Antonio Giorgio ${ }^{[4],}$ Percutaneous needle aspiration is an efficient, effective and low cost technique that can even be performed on an outpatient basis. It is safe, free from significant complications. Percutaneous catheter drainage is preferred in patients in which the patient shows no improvement after $>2$ time percutaneous aspiration $^{[2]}$.

\section{Conclusion}

Percutaneous needle aspiration is preferred in patients with single or multiple liver abscess with cavity size of $>5 \mathrm{~cm}$ and $>50 \mathrm{cc}$ volume. In abscess with cavity size $>10 \mathrm{~cm}$ and single cavity in adults can be treated with Percutaneous catheter drainage.

Both percutaneous aspiration and pigtail catheter drainage are almost equally effective methods for drainage of liver abscess. Although percutaneous aspiration is a simple procedure and can be performed on an outpatient basis, multiple aspirations may be required if the abscess is not completely liquefied. While pigtail catheter drainage is preferred for partially liquefied, single and large abscess, it results in longer duration of hospital stay and poor patient compliance.

\section{References}

1. Singh S, Chaudhary P, Saxena N, Khandelwal S, Poddar DD, Biswal UC. Treatment of liver abscess: prospective randomized comparison of catheter drainage and needle aspiration. Ann Gastroenterol. 2013;26(4):332-339. PMID: 24714320 ; PMCID: PMC3959473.

2. Arpit Bansal, Liver abscess: catheter drainage v/s needle aspiration, Int Surg J. 2015 Feb;2(1):20-25

3. Wang YC, Yang KW, Lee TP, et al. Increased risk of pyogenic liver abscess in patients with alcohol intoxication: A population-based retrospective cohort 
study. Alcohol. 2017;64:23-28.

doi:10.1016/j.alcohol.2017.05.003

4. Giorgio A, de Stefano G, Di Sarno A, Liorre G, Ferraioli G. Percutaneous needle aspiration of multiple pyogenic abscesses of the liver: 13-year single-center experience. AJR Am J Roentgenol. 2006;187(6):1585-1590.

doi:10.2214/AJR.05.1104 